\title{
CHARACTERISTIC INVARIANT OF TENSOR PRODUCT ACTIONS AND ACTIONS ON CROSSED PRODUCTS
}

\author{
YUKAKO MIWA and YOSHIKAZU KATAYAMA
}

(Received 10 May 1999; revised 13 March 2001)

Communicated by G. Willis

\begin{abstract}
The first purpose of this paper is to give a tensor product formula of the characteristic invariant and modular invariant for a tensor product action of a discrete group $G$ on AFD factors. The second purpose is to describe a characteristic invariant and modular invariant of the extended action to a crossed product in terms of the original invariants.
\end{abstract}

2000 Mathematics subject classification: primary $46 \mathrm{~L} 40$.

\section{Introduction}

The cocycle conjugacy class of an action $\alpha$ of a countable discrete amenable group $G$ on an approximately finite dimensional (abbreviated AFD) factor $\mathscr{M}$ was completed in the recent article [11]. This was done by means of the associated characteristic invariant $\chi(\alpha) \in \Lambda\left(G, \alpha^{-1}(\operatorname{Cnt}(\mathscr{H})), \mathrm{H}_{\theta}^{1}(\mathscr{F}(\mathscr{H}))\right)$ and the modular invariant $\nu_{\alpha} \in$ $\operatorname{Hom}_{G}\left(\alpha^{-1}(\operatorname{Cnt}(\mathscr{M})), \mathrm{H}_{\theta}^{1}(\mathscr{F}(\mathscr{M}))\right.$ which is the canonical pullback of the intrinsic invariant of the AFD factor, which is the underlying algebra of the action. These results, due to many mathematicians $[9,10,12,13,14,17,19,20]$, started from the work of Connes [3,6]. A comprehensive account of the subject is presented in the joint work of Katayama, Sutherland and Takesaki cited above. In this article we are concerned with the problem of determining these invariants for tensor product actions and actions on crossed product from those associated with the original action.

In the case that both carrier algebras $\mathscr{M}_{1}$ of $\alpha_{1}$ and $\mathscr{M}_{2}$ of $\alpha_{2}$ are of type $\mathrm{II}_{1}$, the invariants of the tensor product action $\alpha_{1} \otimes \alpha_{2}$, say $\alpha$, on $\mathscr{M}=\mathscr{M}_{1} \otimes \mathscr{M}_{2}$ are almost 
just products of the original ones. So it does not pose any particular difficulty. But in the case that $\mathscr{M}_{1}$ and $\mathscr{M}_{2}$ are not semi-finite, it poses an interesting challenge. For example, the tensor product $\sigma_{1} \otimes \sigma_{2}$ of $\sigma \in \operatorname{Cnt}\left(\mathscr{M}_{1}\right)$ and $\sigma_{2} \in \operatorname{Cnt}\left(\mathscr{M}_{2}\right)$ is not necessarily in $\operatorname{Cnt}\left(\mathscr{M}_{1} \otimes \mathscr{M}_{2}\right)$ which means that $\left(\alpha_{1} \otimes \alpha_{2}\right)^{-1}\left(\operatorname{Cnt}\left(\mathscr{M}_{1} \otimes \mathscr{M}_{2}\right)\right) \neq$ $\alpha_{1}^{-1}\left(\operatorname{Cnt}\left(\mathscr{M}_{1}\right)\right) \cap \alpha_{2}{ }^{-1}\left(\operatorname{Cnt}\left(\mathscr{M}_{2}\right)\right)$. Thus, the basic ingredient $\alpha^{-1}(\operatorname{Cnt}(\mathscr{M}))$ of the characteristic invariant $\chi(\alpha)$ has to be determined based on more data $\left\{\chi\left(\alpha_{1}\right), \nu_{\alpha_{1}}\right\}$ and $\left\{\chi\left(\alpha_{2}\right), \nu_{\alpha_{2}}\right\}$ not just $N_{1}=\alpha_{1}^{-1}\left(\operatorname{Cnt}\left(\mathscr{M}_{1}\right)\right)$ and $N_{2}=\alpha_{2}{ }^{-1}\left(\operatorname{Cnt}\left(\mathscr{M}_{2}\right)\right)$ (See Theorem 2.1). Every III-factor is a crossed product of $\mathrm{II}_{\infty}$-von Neumann algebra $\mathscr{N}$ by dual action $\theta$ of modular automorphism group [21] and the centre $\mathscr{C}$ of $\mathscr{N}$ with an action $\theta$ is called the smooth flow of weight for an AFD III factor. The AFD III factors are classified up to isomorphism by $[5,4,7,15]$. In the case of an AFD factor, it is well known that every centrally trivial automorphism is an extended modular automorphism up to inner automorphism and the canonical extension on $\mathscr{N}$ is also inner $[2,8,13]$. Therefore in the proof of Theorem 2.1 , we deal with automorphisms on $\mathscr{N}$. To show that the tensor product formula is computable, we give a standard form of characteristic invariant and modular invariant in the case of $\operatorname{III}_{\lambda}(0<\lambda<1)$ factors and we propose the tensor product formula of them exactly in this case.

The second purpose is to describe the characteristic invariant and modular invariant of the action, which is extended to a crossed product, in terms of the original invariants. Sekine [18] already gave the smooth flow of weight of the crossed product by making use of the original smooth flow of weight and the invariants of an action. We utilize his frame to define the characteristic invariant of the extended action. Here our problem is also how to define the normal subgroup of $G$ which is a centrally trivial part of the extended action. We characterize this normal subgroup with a cocycle (See Theorem 3.2). Once we characterize it successfully, the computations of the invariants for the extended action are relatively easy. It is shown in Proposition 3.3 that its invariants are computed explicitly in the case of the crossed product of $\mathrm{III}_{\lambda}$ $(0<\lambda \leqq 1)$ factors by discrete abelian group.

The first author would like to express her sincere gratitude to Professor Hisashi Choda for his helpful suggestions and constant encouragement.

\section{Characteristic invariant for the tensor product of actions}

First we give a brief review of the properties of characteristic invariants (see for example [20]).

Let $G$ be a separable locally compact group with a normal subgroup $N$ and $\alpha$ be an action of $G$ on an abelian von Neumann algebra $\mathscr{A}$.

The set $Z_{\alpha}(G, N, \mathscr{U}(\mathscr{A}))$ consists of pairs $(\lambda, \mu)$ such that

$$
\lambda: N \times G \rightarrow \mathscr{U}(\mathscr{A}) \text { and } \mu: N \times N \rightarrow \mathscr{U}(\mathscr{A})
$$


are Borel maps satisfying the following conditions:

$$
\begin{aligned}
& \mu(m, n) \mu(m n, l)=\alpha_{m}(\mu(n, l)) \mu(m, n l), \quad m, n, l \in N ; \\
& \alpha_{g}\left(\lambda\left(g^{-1} n g, h\right)\right) \lambda(n, g)=\lambda(n, g h), \quad m \in N, g, h \in G ; \\
& \lambda(m, m)=\mu\left(m, m^{-1} n m\right) \mu(n, m)^{*}, \quad m, n \in N ; \\
& \lambda(m, g) \alpha_{m}(\lambda(n, g)) \lambda(m n, g)^{*} \\
& \quad=\alpha_{g}\left(\mu\left(g^{-1} n g, g^{-1} m g\right)\right) \mu(n, m)^{*}, \quad m, n \in N, g \in G ; \\
& \mu(m, n)=1 \text { and } \lambda(n, g)=1 \\
& \quad \text { if and only if } m, n \in N, g \in G \text { is the identity. }
\end{aligned}
$$

The set $B_{\alpha}(G, N, \mathscr{U}(\mathscr{A}))$ consists of pairs $\left(\partial_{1} d, \partial_{2} d\right)$, where the map $d: N \rightarrow \mathscr{U}(\mathscr{A})$ is Borel and

$$
\left\{\begin{array}{l}
\left(\partial_{1} d\right)(n, g)=\alpha_{g}\left(d\left(g^{-1} n g\right)\right) d(n)^{*} \\
\left(\partial_{2} d\right)(m, n)=d(m) \alpha_{m}(d(n)) d(m n)^{*}
\end{array}\right.
$$

The quotient group $\Lambda_{\alpha}(G, N, \mathscr{U}(\mathscr{A}))$ is as follows

$$
\Lambda_{\alpha}(G, N, \mathscr{U}(\mathscr{A}))=Z_{\alpha}\left(G, N,(U(\mathscr{A})) / B_{\alpha}(G, N, \mathscr{U}(\mathscr{A})),\right.
$$

and it is called a characteristic invariant for the action $\alpha$. The action $\alpha$ is extended to an action of $G \times \mathbb{R}$ (denoted by the same $\alpha$ ) and $N$ acts trivially on $\mathscr{A}$, and $\mathbb{R}$ acts ergodically on $\mathscr{A}$.

By [20, Theorem 2.2], we have a natural exact sequence

$$
\begin{aligned}
\Lambda_{\alpha}(G \times \mathbb{R}, N, \mathscr{U}(\mathscr{A})) & \rightarrow \Lambda_{\alpha}(G, N, \mathscr{U}(\mathscr{A}))^{\mathbb{R}} \times \operatorname{Hom}_{G}\left(N, \mathrm{H}_{\alpha}^{1}(\mathbb{R}, \mathscr{U}(\mathscr{A}))\right) \\
& \stackrel{\delta}{\rightarrow} \mathrm{H}_{\alpha}^{1}\left(\mathbb{R}, B_{\alpha}(G, N, \mathscr{U}(\mathscr{A}))\right) .
\end{aligned}
$$

For $\chi=[\lambda, \mu] \in \Lambda_{\alpha}(G \times \mathbb{R}, N, \mathscr{U}(\mathscr{A}))$, a restricted characteristic invariant $\left[\left.\lambda\right|_{N \times G}, \mu\right]$ on $G$ is an element of $\Lambda_{\alpha}(G, N, \mathscr{U}(\mathscr{A}))^{\mathbb{R}}$ and the map $c: n \in N \rightarrow$ $\left.\lambda\right|_{N \times \mathbb{R}}(n, \cdot)=c(n)(\cdot)$ induces a map $v: n \in N \rightarrow[c(n)] \in \mathrm{H}_{\alpha}^{1}(\mathbb{R}, \mathscr{U}(\mathscr{A}))$ which is a $G$-equivariant homamorphism. This is called a modular invariant. For $\chi=[\lambda, \mu] \in \Lambda_{\alpha}(G, N, \mathscr{U}(\mathscr{A}))^{\mathbb{R}}$, we define

$$
\left\{\begin{array}{l}
\tilde{\lambda}(t, n, g)=\alpha_{t}(\lambda) \lambda^{*}(n, g) ; \\
\tilde{\mu}(t, n, g)=\alpha_{t}(\mu) \mu^{*}(m, n), \quad(t \in \mathbb{R}, m, n \in N, \text { and } g \in G),
\end{array}\right.
$$

and

$$
\left\{\begin{array}{l}
\delta_{1}: \chi \in \Lambda_{\alpha}(G, N, \mathscr{U}(\mathscr{A}))^{\mathbb{R}} \rightarrow \delta_{1}(\chi)=[\tilde{\lambda}, \tilde{\mu}] \in \mathrm{H}_{\alpha}^{1}\left(\mathbb{R}, B_{\alpha}(G, N, \mathscr{U}(\mathscr{A}))\right) ; \\
\delta_{2}: v \in \operatorname{Hom}_{G}\left(N, \mathrm{H}_{\alpha}^{1}(\mathbb{R}, \mathscr{U}(\mathscr{A}))\right) \\
\quad \rightarrow \delta_{2}(\nu)=\left[\partial_{1} c, \partial_{2} c\right] \in \mathrm{H}_{\alpha}^{1}\left(\mathbb{R}, B_{\alpha}(G, N, \mathscr{U}(\mathscr{A}))\right),
\end{array}\right.
$$


where the map $c: n \in N \rightarrow c(n) \in Z_{\alpha}^{1}(\mathbb{R}, \mathscr{U}(\mathscr{A}))$ is a Borel map lifting $v$. For $(\chi, \nu) \in \Lambda_{\alpha}(G, N, \mathscr{U}(\mathscr{A}))^{\mathbb{R}} \times \operatorname{Hom}_{G}\left(N, \mathrm{H}_{\alpha}^{1}(\mathbb{R}, \mathscr{U}(\mathscr{A}))\right)$, we define

$$
\delta(\chi, v)=\delta_{1}(\chi)-\delta_{2}(v)
$$

We remark that by [20, Lemma 2.1], for $t \in \mathbb{R}, g \in G$,

$$
\left\{\begin{array}{l}
\alpha_{t}(\lambda) \lambda^{*}(n, g)=\alpha_{g}\left(\lambda\left(g^{-1} n g, t\right)\right) \lambda(n, t)^{*} ; \\
\alpha_{t}(\mu) \mu^{*}(m, n)=\lambda(m, t) \alpha_{m}(\lambda(n, t)) \lambda(m n, t)^{*}
\end{array}\right.
$$

for $\lambda \in Z_{\alpha}(G \times \mathbb{R}, N, \mathscr{U}(\mathscr{A}))$.

From now on, we assume that the group $G$ is discrete. We consider a tensor product of two actions of $G$ on AFD factors of type III. Our aim is to show that the characteristic invariant and the modular invariant for the tensor product of two actions can be expressed by (2.4) and (2.5). We give an example in which its invariants can be computed explicitly.

Let $\mathscr{M}$ be approximately finite dimensional (AFD) factor of type III and $\alpha$ be an action of $G$ on $\mathscr{M}$. We may suppose that the action $\alpha$ admits an invariant dominant weight $\varphi$ on $\mathscr{M}$. A dual action $\theta_{t}$ of the modular automorphism $\sigma^{\varphi}$ associated with $\varphi$ is defined on a crossed product $\mathscr{N}=\mathscr{M} \rtimes_{\sigma^{\varphi}} \mathbb{R}$ by

$$
\theta_{l}\left(\pi_{\varphi}(x)\right)=\pi_{\varphi}(x), \quad \theta_{l}\left(\lambda_{\varphi}(s)\right)=e^{-i t s} \lambda_{\varphi}(s),
$$

where $x \in \mathscr{M}$ and $t, s \in \mathbb{R}$ and the set $\left\{\pi_{\varphi}(x), \lambda_{\varphi}(s): x \in \mathscr{M}, s \in \mathbb{R}\right\}$ generates $\mathscr{N}$. Thanks to Connes' Radon-Nikodým cocycle [1], the isomorphic class of the crossed product $\mathscr{M} \rtimes_{\sigma^{\bullet}} \mathbb{R}$ is independent of the choice of weights. For an automorphism $\gamma \in \operatorname{Aut}(\mathscr{M})$, we can extend canonically an automorphism $\tilde{\gamma} \in \operatorname{Aut}(\mathscr{N})$

$$
\begin{cases}\tilde{\gamma}\left(\pi_{\varphi}(x)\right)=\pi_{\varphi}(\gamma(x)) & \text { for } x \in \mathscr{M} ; \\ \tilde{\gamma}\left(\lambda_{\varphi}(s)\right)=\pi_{\varphi}\left(\left(D \varphi \gamma^{-1}: D \varphi\right)_{s}\right) \lambda_{\varphi}(s) & \text { for } s \in \mathbb{R},\end{cases}
$$

where $\left(D \varphi \gamma^{-1}: D \varphi\right)_{s}$ is Connes' cocycle [7,8]. The centre $\mathscr{C}$ of $\mathscr{N}$ is isomorphic to a smooth flow of weight for $\mathscr{M}$ and the restricted action $\theta_{t}$ on $\mathscr{C}$ is called a flow. Let $\alpha$ be an action of $G$ on $\mathscr{M}$. The restricted action $\tilde{\alpha}_{g}$ on $\mathscr{C}$ is just $\bmod \alpha_{g}$ which is called the module. We sometimes denote the above restricted actions by the same symbol $\theta_{t}$ and $\alpha_{g}$.

The definition of characteristic invariant and modular invariant for the action $\alpha$ on flow of type III are found in [20] or [13]. Here we give definitions which are equivalent to the original ones in [20]. Let $N=N_{\alpha}$ be a normal subgroup of $G$ defined by

$$
N_{\alpha}=\left\{n \in G: \tilde{\alpha}_{n}=\operatorname{Ad} u(\alpha)_{n} \text { for some } u(\alpha)_{n} \in \mathscr{U}(\mathscr{N})\right\}
$$


and the unitary $u_{n}=u(\alpha)_{n}$ yields a characteristic invariant $\Lambda(\alpha)=[\lambda, \mu] \epsilon$ $\Lambda_{\alpha}(G, N, \mathscr{U}(\mathscr{C}))$ and a modular invariant $\nu_{\alpha}=[c(n)] \in \operatorname{Hom}_{G}\left(N, \mathrm{H}_{\theta}^{1}(\mathbb{R}, \mathscr{U}(\mathscr{C}))\right)$ for the action $\alpha$ as follows:

$$
\left\{\begin{array}{l}
\tilde{\alpha}_{g}\left(u_{g^{-1} n g}\right)=\lambda(n, g) u_{n} \\
u_{m} u_{n}=\mu(m, n) u_{m n} \\
\theta_{t}\left(u_{n}\right)=c(n)(t) u_{n}
\end{array}\right.
$$

where $n, m \in N, g \in G$ and $t \in \mathbb{R}$.

Let $\mathscr{M}_{1}$ and $\mathscr{M}_{2}$ be AFD factors of type III and $\alpha$ and $\beta$ be actions of $G$ on $\mathscr{M}_{1}$ and $\mathscr{M}_{2}$ respectively. With notation as above for each $\mathscr{M}_{1}$ and $\mathscr{M}_{2}$, we define crossed products $\mathscr{N}_{1}=\mathscr{M}_{1} \rtimes_{\sigma^{\omega}} \mathbb{R}$ and $\mathscr{N}_{2}=\mathscr{M}_{2} \rtimes_{\sigma^{\star}} \mathbb{R}$ for invariant dominant weights $\varphi$ and $\psi$. The action $\alpha$ and $\beta$ can be extended to action $\tilde{\alpha}_{g}$ and $\tilde{\beta}_{g}$ on $\mathscr{N}_{1}$ and $\mathscr{N}_{2}$. Moreover $\tilde{\alpha}_{g}$ and $\tilde{\beta}_{g}$ commute with each dual action $\theta_{t}^{1}$ and $\theta_{t}^{2}$, which are denoted by $\alpha_{t}$ and $\beta_{t}$ respectively. We denote a product action $\tilde{\alpha}_{g} \theta_{t}^{1}$ of $G \times \mathbb{R}$ on $\mathscr{N}_{1}$ by $\alpha_{(g, t)}$ without any confusion. Similarly, we define $\beta_{(g, t)}=\tilde{\beta}_{g} \theta_{t}^{2}$. It is easy to check that the crossed product $\mathscr{N}_{3}=\left(\mathscr{M}_{1} \otimes \mathscr{M}_{2}\right) \rtimes_{\sigma^{\varphi \otimes \psi}} \mathbb{R}$ is isomorphic to a subalgebra $\left(\mathscr{M}_{1} \otimes \mathscr{M}_{2}\right) \rtimes_{\sigma^{\varphi} \otimes \sigma^{\psi}}\{(t, t): t \in \mathbb{R}\}$ of $\left(\mathscr{M}_{1} \rtimes_{\sigma^{\psi}} \mathbb{R}\right) \otimes\left(\mathscr{M}_{2} \rtimes_{\sigma^{\psi}} \mathbb{R}\right)$. By the Galois correspondence [21, Theorem 7.2], the von Neumann algebra $\mathscr{N}_{3}$ is isomorphic to the fixed point algebra $\left\{y \in \mathscr{N}_{1} \otimes \mathscr{N}_{2}: \alpha_{t} \otimes \beta_{-t}(y)=y\right\}$, which is identified with $\mathscr{N}_{3}$. The smooth flow of weight $\mathscr{C}_{3}=Z\left(\mathscr{N}_{3}\right)$ for $\mathscr{M}_{1} \otimes \mathscr{M}_{2}$ is isomorphic to $\left(\left\{y \in \mathscr{C}_{1} \otimes \mathscr{C}_{2}: \alpha_{t} \otimes \beta_{-t}(y)=y, t \in \mathbb{R}\right\}, \alpha_{t} \otimes \imath\right)$, where $\mathscr{C}_{i}=Z\left(\mathscr{N}_{i}\right)$.

Let $\chi_{1}=\left[\lambda_{1}, \mu_{1}\right]$ and $\chi_{2}=\left[\lambda_{2}, \mu_{2}\right]$ be characteristic invariants in $\Lambda_{\alpha}\left(G, N_{1}\right.$, $\left.\mathscr{U}\left(\mathscr{C}_{1}\right)\right)$ and $\Lambda_{\beta}\left(G, N_{2}, \mathscr{U}\left(\mathscr{C}_{2}\right)\right)$ associated with the actions $\alpha$ and $\beta$ and $c_{i}(n)(t)$, $(i=1,2)$ be their modular invariants. We identify $\mathscr{C}_{3}$ with $\left\{y \in \mathscr{C}_{1} \otimes \mathscr{C}_{2}: \alpha_{t} \otimes\right.$ $\left.\beta_{-t}(y)=y, t \in \mathbb{R}\right\}$. We define a normal subgroup $N_{3}$ of $G$ and $\lambda_{3}(n, g), \mu_{3}(m, n) \in$ $\mathscr{C}_{3}$ by

$$
\left\{\begin{array}{rr}
N_{3}=\left\{n \in N_{1} \cap N_{2}: c_{1}(n, t) \otimes c_{2}(n,-t)=d_{n}\left(\alpha, \otimes \beta_{-l}\right)\left(d_{n}^{*}\right),\right. & \text { for some } \left.d_{n} \in \mathscr{U}\left(\mathscr{C}_{1} \otimes \mathscr{C}_{2}\right)\right\} ; \\
\lambda_{3}(n, g)=\lambda_{1}(n, g) \otimes \lambda_{2}(n, g)\left(\tilde{\alpha}_{g} \otimes \tilde{\beta}_{g}\right)\left(d_{g^{-1} n g}\right) d_{n}^{*}, & g \in G ; \\
\mu_{3}(m, n)=d_{m n}^{*} d_{m} d_{n} \mu_{1}(m, n) \otimes \mu_{2}(m, n), & m, n \in N_{3} .
\end{array}\right.
$$

We also define, for $n \in N_{3}, t \in \mathbb{R}$,

$$
c_{3}(n, t)=\left(\alpha_{t} \otimes \imath\right)\left(d_{n}\right) d_{n}^{*}\left(c_{1}(n, t) \otimes 1\right),
$$

where $c_{1}(n, t) \otimes c_{2}(n,-t)=d_{n}\left(\alpha_{t} \otimes \beta_{-t}\right)\left(d_{n}^{*}\right)$. Using

$$
\left(\alpha_{t} \otimes \imath\right)\left(d_{n}^{*}\right)=\left(\iota \otimes \beta_{t}\right)\left(d_{n}^{*}\right)\left(c_{1}(n, t) \otimes \beta_{l}\left(c_{2}(n,-t)\right)\right),
$$


it is easy to check that $c_{3}(n, t)=\left(\iota \otimes \beta_{t}\right)\left(d_{n}\right) d_{n}^{*}\left(1 \otimes c_{2}(n, t)\right)$.

It was shown in [16], by an algebraic method, that the tensor product of invariants is well defined and it satisfies the conditions (1)-(5) of characteristic invariant and modular invariant with $\delta\left(\left[\lambda_{3}, \mu_{3}\right],\left[c_{3}\right]\right)=0$. The proof is valid even when the real field $\mathbb{R}$ is replaced by another locally compact group. Here we shall prove the tensor product formula of invariants in the operator algebraic way.

THEOREM 2.1 (Tensor product formula). With notation as above, the characteristic invariant and modular invariant for the product action $\alpha \otimes \beta$ of $G$ on $\mathscr{M}_{1} \otimes \mathscr{M}_{2}$ is $\left[\lambda_{3}, \mu_{3}\right] \in \Lambda_{\alpha \otimes \beta}\left(G, N_{3}, \mathscr{U}\left(\mathscr{C}_{3}\right)\right)^{\mathbb{R}}$ and $\left[c_{3}(n, \cdot)\right] \in \operatorname{Hom}_{G}\left(N_{3}, \mathrm{H}_{\alpha \otimes i}^{1}\left(\mathbb{R}, \mathscr{U}\left(\mathscr{C}_{3}\right)\right)\right)$, where $\lambda_{3}, \mu_{3}$ and $c_{3}$ are derived in (2.4) and (2.5) from the invariants $\left(\lambda_{i}, \mu_{i}\right)$ and $v_{i}=\left[c_{i}(n)\right],(i=1,2)$ for the actions $\alpha$ and $\beta$ on $\mathscr{M}_{1}$ and $\mathscr{M}_{2}$ respectively.

PROOF. By (2.2) we have that $\widetilde{\alpha \otimes \beta}$ on $\mathscr{N}_{3}$ is the restriction of $\tilde{\alpha} \otimes \tilde{\beta}$ on $\mathscr{N}_{3} \subset$ $\mathscr{N}_{1} \otimes \mathscr{N}_{2}$. For $n \in N_{\alpha \otimes \beta}$, take $U_{n} \in \mathscr{N}_{3}$ such that $\left(\tilde{\alpha}_{n} \otimes \tilde{\beta}_{n}\right)(x)=\operatorname{Ad} U_{n}(x)$ for $x \in \mathscr{N}_{3}$. By $[3,5,13]$, the element $n$ is contained in $N_{\alpha} \cap N_{\beta}$. Therefore, we have

$$
\left(\tilde{\alpha}_{n} \otimes \tilde{\beta}_{n}\right)(x)=\operatorname{Ad} u(\alpha)_{n} \otimes u(\beta)_{n}(x)
$$

for $x \in \mathscr{N}_{1} \otimes \mathscr{N}_{2}$ and

$$
\operatorname{Ad} U_{n}(x)=\operatorname{Ad} u(\alpha)_{n} \otimes u(\beta)_{n}(x)
$$

for $x \in \mathscr{N}_{3}$. It follows from $\mathscr{N}_{3} \supset \mathscr{M}_{1} \otimes \mathscr{M}_{2}$ and [11, Lemma 1.1] that there exists $d_{n} \in \mathscr{U}\left(\mathscr{C}_{1} \otimes \mathscr{C}_{2}\right)$ such that $U_{n}=d_{n}\left(u(\alpha)_{n} \otimes u(\beta)_{n}\right)$. Since $\left(\alpha_{t} \otimes \beta_{-t}\right)\left(U_{n}\right)=U_{n}$ for $t \in \mathbb{R}$, we have, by (2.3),

$$
\begin{aligned}
d_{n}\left(u(\alpha)_{n} \otimes u(\beta)_{n}\right) & =\left(\alpha_{t} \otimes \beta_{-t}\right)\left(d_{n}\right) \alpha_{t}\left(u(\alpha)_{n}\right) \otimes \beta_{-t}\left(u(\beta)_{n}\right) \\
& =\left(\alpha_{t} \otimes \beta_{-t}\right)\left(d_{n}\right) c_{1}(n, t) u(\alpha)_{n} \otimes c_{2}(n,-t) u(\beta)_{n},
\end{aligned}
$$

which implies that $d_{n} \alpha_{t} \otimes \beta_{-t}\left(d_{n}^{*}\right)=c_{1}(n, t) \otimes c_{2}(n,-t)$.

Conversely, suppose that for $n \in N_{\alpha} \cap N_{\beta}$, there is some $d_{n} \in \mathscr{U}\left(\mathscr{C}_{1} \otimes \mathscr{C}_{2}\right)$ such that $d_{n}\left(\alpha_{t} \otimes \beta_{-l}\right)\left(d_{n}^{*}\right)=c_{1}(n, t) \otimes c_{2}(n,-t)$. We set $U_{n}$ by $d_{n}\left(u(\alpha)_{n} \otimes u(\beta)_{n}\right)$. Then

$$
\operatorname{Ad} U_{n}(x)=\operatorname{Ad} d_{n}\left(\tilde{\alpha}_{n} \otimes \tilde{\beta}_{n}\right)(x)=\left(\tilde{\alpha}_{n} \otimes \tilde{\beta}_{n}\right)(x)
$$

for $x \in \mathscr{N}_{1} \otimes \mathscr{N}_{2}$. Moreover, since we compute

$$
\begin{aligned}
\left(\alpha_{t} \otimes \beta_{-t}\right)\left(U_{n}\right) & =\left(\alpha_{t} \otimes \beta_{-t}\right)(d) c_{1}(n, t) u(\alpha)_{n} \otimes c_{2}(n,-t) u(\beta)_{n} \\
& =d_{n}\left(u(\alpha)_{n} \otimes u(\beta)_{n}\right)=U_{n},
\end{aligned}
$$

the unitary $U_{n}$ is in $\mathscr{N}_{3}$. We have shown that

$$
\begin{aligned}
N_{\alpha \otimes \beta}=\left\{n \in N_{\alpha} \cap N_{\beta}: d_{n}\left(\alpha_{t} \otimes \beta_{-t}\right)\left(d_{n}^{*}\right)=\right. & c_{1}(n, t) \otimes \\
& \text { for some } d_{n} \in \mathscr{U}(n,-t) \\
&
\end{aligned}
$$


Using (2.3) we obtain

$$
\begin{aligned}
\lambda_{3}(n, g) & =\left(\tilde{\alpha}_{g} \otimes \tilde{\beta}_{g}\right)\left(U_{g^{-1} n g}\right) U_{n}^{*} \\
& =\left(\tilde{\alpha}_{g} \otimes \tilde{\beta}_{g}\right)\left(d_{g^{-1} n g}\right)\left(\tilde{\alpha}_{g}(u(\alpha))_{g^{-1} n g}\right) \otimes \tilde{\beta}_{g}\left(u(\beta)_{g^{-1} n g}\right)\left(u(\alpha)_{n}^{*} \otimes u(\beta)_{n}^{*}\right) d_{n}^{*} \\
& =\left(\tilde{\alpha}_{g} \otimes \tilde{\beta}_{g}\right)\left(d_{g^{-1} n g}\right) d_{n}^{*} \lambda_{1}(n, g) \otimes \lambda_{2}(n, g) \\
\mu_{3}(m, n) & =U_{m} U_{n} U_{m n}^{*}=d_{m n}^{*} d_{n} d_{m}\left(\mu_{1}(m, n) \otimes \mu_{2}(m, n)\right) \\
c_{3}(n, t) & =\left(\alpha_{t} \otimes \imath\right)\left(U_{n}\right) U_{n}^{*}=\left(\alpha_{t} \otimes \imath\right)\left(d_{n}\right) d_{n}^{*}\left(c_{1}(n, t) \otimes 1\right)
\end{aligned}
$$

In the case of $\mathrm{III}_{0}$-factors, the tensor product formula of characteristic invariant and modular invariant depends heavily on the flow of weights and we cannot give its formula explicitly. We give a standard form of characteristic invariant and modular invariant in the case of III $_{\lambda}$-factors $(0<\lambda<1)$ and we show the tensor product formula of them exactly.

Let $\mathscr{M}$ be a factor of type $\operatorname{III}_{\lambda}(0<\lambda \leqq 1)$. It is well known that the flow of weight $\left(\mathscr{F}(\mathscr{M}), F^{\mathscr{M}}\right)=\left(\mathscr{C}, \theta_{t}\right)$ is regarded as $\left(L^{\infty}([0,-\log \lambda))\right.$, translation by $\left.-t\right)$ and the cohomology group $\mathrm{H}_{\theta}^{1}(\mathbb{R}, \mathscr{U}(\mathscr{C}))$ is as follows

$$
\mathrm{H}_{\theta}^{1}(\mathbb{R}, \mathscr{U}(\mathscr{C})) \cong \begin{cases}\left\{e^{-i t s} ; s \in \mathbb{R} / T \mathbb{Z}\right\} & (0<\lambda<1) \\ \left\{e^{-i t s} ; s \in \mathbb{R}\right\} & (\lambda=1),\end{cases}
$$

where $T=-2 \pi / \log \lambda$. We may choose the modular invariant $[c(n)] \in \mathrm{H}_{\theta}^{1}(\mathbb{R}, \mathscr{U}(\mathscr{C}))$ to be of the form $c(n)(t)=e^{i t v(n)}$, where $v(n) \in[0, T)$. We identify the real number $v(n)$ with the modular invariant $v(n)=[c(n)] \in \mathrm{H}_{\theta}^{1}(\mathbb{R}, \mathscr{U}(\mathscr{C}))$. The following lemma was proved in [20], we include here a brief proof.

LEMMA 2.2. Let $\alpha$ be an action of $G$ on AFD factor $\mathscr{M}$ of type $\operatorname{III}_{\lambda}(0<\lambda<1)$. The characteristic invariant $[\lambda, \mu]$ of $\alpha$ is of the form (up to cohomology)

$$
\left\{\begin{array}{l}
\lambda(n, g)(w) \text { is a constant function; } \\
\mu(m, n)(w)=\bar{\mu}(m, n) e^{i w(\nu(m n)-v(m)-v(n))} .
\end{array}\right.
$$

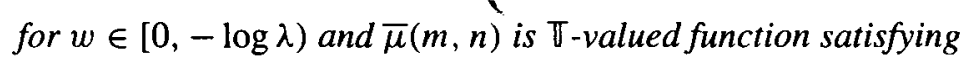

$$
\left\{\begin{array}{l}
\bar{\mu}(m, n) \bar{\mu}(m n, l)=\bar{\mu}(n, l) \bar{\mu}(m, n l) \\
\lambda(m, g) \lambda(n, g) \overline{\lambda(m n, g)}=\bar{\mu}\left(g^{-1} m g, g^{-1} n g\right) \overline{\bar{\mu}(m, n)} e^{-i \tau(g)(\nu(m n)-\nu(m)-v(n))},
\end{array}\right.
$$

where $\bmod \alpha_{g}(f)(w)=f(w-\tau(g))$, for $f \in L^{\infty}([0,-\log \lambda))$, where $\tau(g) \in[0,-\log \lambda)$.

PROOF. We may assume $c(n)(t)=e^{i n(n)}$. By $(2.1)$ and $v\left(g^{-1} n g\right)=v(n)$, we have

$$
\alpha_{t}(\lambda) \lambda^{*}(n, g)=\tilde{\alpha}_{g}\left(c\left(g^{-1} n g, t\right)\right) c(n, t)^{*}=e^{i t\left(v\left(g^{-1} n g\right)-v(n)\right)}=1 .
$$


Since $\alpha_{t}$ is ergodic, the unitary $\lambda(n, g)$ must be constant. By the Fourier expansion of $\mu, \mu(m, n)(w)=\sum_{k} a_{k} e^{i w k(2 \pi / \log \lambda)}$, where $a_{k} \in \mathbb{C}$. By (2.1), we have

$$
\alpha_{t}(\mu) \mu^{*}(m, n)=c(m, t) c(n, t) c(m n, t)^{*}=e^{i t(v(m)+\nu(n)-v(m n))} .
$$

By comparison of the Fourier coefficients, we get

$$
a_{k} e^{-i(k 2 \pi / \log \lambda}=e^{i t(\nu(m)+\nu(n)-\nu(m n))} a_{k}
$$

for $k \in \mathbb{Z}$. Then there exists a unique $k(2 \pi / \log \lambda)=v(m n)-v(m)-v(n)$ such that $a_{k} \neq 0$. Therefore, $\mu(m, n)$ is of the form $\bar{\mu}(m, n) e^{i w(\nu(m n)-v(m)-\nu(n))}$, where $\bar{\mu}(m, n)$ is scalar. The statement (2.7) follows from conditions (1) and (4).

Let $\mathscr{M}_{1}$, and $\mathscr{M}_{2}$ be AFD factors of type $\operatorname{III}_{\lambda_{1}}$ and $\operatorname{III}_{\lambda_{2}}\left(0<\lambda_{1}, \lambda_{2} \leqq 1\right)$, and $\alpha$ and $\beta$ be actions of the group $G$ on $\mathscr{M}_{1}$ and $\mathscr{M}_{2}$ respectively. We remark that the following lemma is related to [13, Lemma 1.7].

LEMMA 2.3. Let $\nu_{i}$ be the modular invariants for $\alpha$ and $\beta$, where $v_{i}(n) \in\left[0, T_{i}\right)$ and $T_{i}=-2 \pi / \log \lambda_{i}$ respectively $(i=1,2)$.

(1) If $\mathscr{M}_{1}$ and $\mathscr{M}_{2}$ are of type $\mathrm{III}_{\lambda_{1}}$ and $\mathrm{III}_{\lambda_{2}}$ with $0<\lambda_{1}, \lambda_{2}<1$, there is an operator $d \in L^{\infty}\left(\left[0,-\log \lambda_{1}\right) \times\left[0,-\log \lambda_{2}\right)\right)$ such that

$$
\alpha_{t} \otimes \beta_{t}\left(d^{*}\right) d=c_{1}(n)(t) \otimes c_{2}(n)(-t)=e^{i t\left(v_{1}(n)-v_{2}(n)\right)}
$$

if and only if there exists $\left(k_{1}(n), k_{2}(n)\right) \in \mathbb{Z}^{2}$ such that

$$
v_{1}(n)+k_{1}(n) T_{1}=\nu_{2}(n)+k_{2}(n) T_{2} .
$$

Moreover, the operator $d$ can be chosen to be of the form

$$
d\left(w_{1}, w_{2}\right)=e^{-i\left(w_{1} k_{1}(n) T_{1}+w_{2} k_{2}(n) T_{2}\right)}
$$

for $\left(w_{1}, w_{2}\right) \in\left[0,-\log \lambda_{1}\right) \times\left[0,-\log \lambda_{2}\right)$.

(2) If $\mathscr{M}_{1}$ is of type $\mathrm{III}_{\lambda_{1}}$ with $0<\lambda_{1}<1$ and $\mathscr{M}_{2}$ is of type $\mathrm{III}_{1}$, we may replace the condition (2.9) and the operator $d$ in (2.10) by

$$
\begin{aligned}
& v_{1}(n)+k_{1}(n) T_{1}=\nu_{2}(n) ; \\
& d\left(w_{1}\right)=e^{-i w_{1} k_{1}(n) r_{1}} .
\end{aligned}
$$

PROOF. (1) The operator $d$ is expressed by the Fourier expansion

$$
d\left(w_{1}, w_{2}\right)=\sum_{k, m} a_{k, m} e^{-i w_{1} k T_{1}} \times e^{-i w_{2} m T_{2}} .
$$


We compute

$$
\left\{\begin{aligned}
\alpha_{t} \otimes \beta_{-t}(d) & =\sum_{k, m} a_{k, m} e^{-i\left(w_{1}-t\right) k T_{1}} \times e^{-i\left(w_{2}+t\right) m T_{2}} \\
& =\sum_{k, m} a_{k, m} e^{i t\left(k T_{1}-m T_{2}\right)} e^{-i w_{1} k T_{1}} \times e^{-i w_{2} m T_{2}} \\
e^{-i t\left(v_{1}(n)-v_{2}(n)\right)} d & =\sum_{k, m} e^{-i t\left(v_{1}(n)-v_{2}(n)\right)} a_{k, m} e^{-i w_{1} k T_{1}} \times e^{-i w_{2} m T_{2}}
\end{aligned}\right.
$$

By (2.8), there exists $\left(k_{1}(n), k_{2}(n)\right) \in \mathbb{Z}^{2}$ such that

$$
\left\{\begin{array}{l}
a_{k_{1}(n), k_{2}(n)} \neq 0 \\
v_{1}(n)-v_{2}(n)=-k_{1}(n) T_{1}+k_{2}(n) T_{2}
\end{array}\right.
$$

Conversely, take a function $d\left(w_{1}, w_{2}\right)$ as follows $d\left(w_{1}, w_{2}\right)=e^{-i\left(w_{1} k_{1}(n) T_{1}+w_{2} k_{2}(n) T_{2}\right)}$, then by the condition (2.9), we conclude

$$
\begin{aligned}
\alpha_{t} \otimes \beta_{-t}\left(d^{*}\right) d & =e^{i\left(\left(w_{1}-t\right) k_{1}(n) T_{1}+\left(w_{2}+t\right) k_{2}(n) T_{2}\right)} e^{-i\left(w_{1} k_{1}(n) T_{1}+w_{2} k_{2}(n) T_{2}\right)} \\
& =e^{-i t\left(k_{1}(n) T_{1}-k_{2}(n) T_{2}\right)}=e^{i t\left(\nu_{1}(n)-v_{2}(n)\right)}
\end{aligned}
$$

(2) If $\mathscr{M}_{2}$ is of type $\mathrm{III}_{1}$, the smooth flow of weight $\mathscr{M}_{2}$ is trivial. Therefore the operator $d$ is a function on $\left[0,-\log \lambda_{1}\right)$. The statements in (2) can be shown by repeating the argument of (1).

If the invariants $\left(\lambda_{i}, \mu_{i}\right)$ are of the form (2.6) for $i=1,2$, then we compute $c_{3}, \lambda_{3}$ and $\mu_{3}$ using the definition, with the function $d_{n}=d$ in (2.10)

$$
\begin{aligned}
c_{3}(n, t)= & \left(\alpha_{t} \otimes l\right)\left(d_{n}\right) d_{n}^{*}\left(c_{1}(n, t) \otimes 1\right) \\
= & e^{-i\left(\left(w_{1}-t\right) k_{1}(n) T_{1}+w_{2} k_{2}(n) T_{2}\right)} e^{i\left(w_{1} k_{1}(n) T_{1}+w_{2} k_{2}(n) T_{2}\right)} e^{i t v_{1}(n)} \\
= & e^{i t\left(v_{1}(n)+k_{1}(n) T_{1}\right)} \\
\lambda_{3}(n, g)= & \left(\tilde{\alpha}_{g} \otimes \tilde{\beta}_{g}\right)\left(d_{g^{-1} n g}\right) d_{n}^{*}\left(\lambda_{1}(n, g) \otimes \lambda_{2}(n, g)\right) \\
= & \lambda_{1}(n, g) \lambda_{2}(n, g) e^{i\left(\tau_{1}(g) k_{1}\left(g^{-1} n g\right) T_{1}+\tau_{2}(g) k_{2}\left(g^{-1} n g\right) T_{2}\right)} \\
& \times e^{-i w_{1}\left(k_{1}\left(g^{-1} n g\right)-k_{1}(n)\right) T_{1}} e^{-i w_{2}\left(k_{2}\left(g^{-1} n g\right)-k_{2}(n)\right) T_{2}} ; \\
\mu_{3}(m, n)= & d_{m n}^{*} d_{m} d_{n}\left(\mu_{1}(m, n) \otimes \mu_{2}(m, n)\right) \\
= & \overline{\mu_{1}}(m, n) \overline{\mu_{2}}(m, n) e^{i w_{1}\left(v_{1}(m n)-v_{1}(m)-v_{1}(n)\right)} e^{i w_{2}\left(v_{2}(m n)-v_{2}(m)-v_{2}(n)\right.} \\
& \times e^{i w_{1}\left(k_{1}(m n)-k_{1}(m)-k_{1}(n)\right) T_{1}} e^{\left.i w_{2}\left(k_{2}(m n)-k_{2}(m)-k_{2}(n)\right) T_{2}\right)} .
\end{aligned}
$$


In the case when $\mathscr{M}_{2}$ is of type $\mathrm{III}_{1}$, we can take $d_{n}$ as in $\left(2.10^{\prime}\right)$. Then

$$
\left\{\begin{aligned}
c_{3}(n, t)= & e^{i t\left(\nu_{1}(n)+k_{1}(n) T\right)} \\
\lambda_{3}(n, t)= & \lambda_{1}(n, g) \lambda_{2}(n, g) e^{i \tau_{1}(g) k_{1}\left(g^{-1} n g\right) T_{1}} e^{-i w_{1}\left(k_{1}\left(g^{-1} n g\right)-k_{1}(n)\right) T_{1}} \\
\mu_{3}(m, n)= & \overline{\mu_{1}}(m, n) \overline{\mu_{2}}(m, n) \\
& \times e^{i w_{1}\left(\nu_{1}(m n)-\nu_{1}(m)-\nu_{1}(n)\right)} e^{i w_{1}\left(k_{1}(m n)-k_{1}(m)-k_{1}(n)\right) T_{1}}
\end{aligned}\right.
$$

If $\log \lambda_{2} / \log \lambda_{1}$ is rational with $\log \lambda_{2} / \log \lambda_{1}=l_{2} / l_{1}$ simple fraction $\left(l_{2}, l_{1} \in \mathbb{N}\right)$, then we set $\lambda_{3}=\lambda_{2}^{1 / l_{2}}=\lambda_{1}^{1 / l_{1}}$, and the tensor product factor $\mathscr{M}_{1} \otimes \mathscr{M}_{2}$ is of type III $_{\lambda_{3}}$. We set

$$
\nu_{3}(n)=\left(k_{1}(n) T_{1}+\nu_{1}(n)\right)-\left[\left(k_{1}(n) T_{1}+\nu_{1}(n)\right) / T_{3}\right] T_{3} \in\left[0, T_{3}\right)
$$

where $T_{3}=-2 \pi / \log \lambda_{3}$ and [ $\left.\cdot\right]$ is the Gauss symbol. If $\log \lambda_{2} / \log \lambda_{1}$ is irrational or $\lambda_{2}=1$, then $\mathscr{M}_{1} \otimes \mathscr{M}_{2}$ is of type $\mathrm{III}_{1}$. Hence we set

$$
v_{3}(n)=k_{1}(n) T_{1}+v_{1}(n) \in \mathbb{R} .
$$

PROPOSITION 2.4. (1) If $\log \lambda_{2} / \log \lambda_{1}$ is rational, then the characteristic invariant $\left(\lambda_{3}, \mu_{3}\right)$ for the product action $\alpha \otimes \beta$ of $G$ on $\mathscr{M}_{1} \otimes \mathscr{M}_{2}$ is cohomologous to

$$
\left\{\begin{array}{l}
\lambda_{1}(n, g) \lambda_{2}(n, g) e^{i\left(\left(\tau_{1}(g)+\tau_{2}(g)\right) \nu_{3}\left(g^{-1} n g\right)-\tau_{1}(g) \nu_{1}\left(g^{-1} n g\right)-\tau_{2}(g) \nu_{2}\left(g^{-1} n g\right)\right)} \\
\overline{\mu_{1}}(m, n) \overline{\mu_{2}}(m, n) e^{i w\left(\nu_{3}(m n)-\nu_{3}(m)-v_{3}(n)\right)}
\end{array}\right.
$$

for $w \in\left[0,-\log \lambda_{3}\right)$.

(2) If $\log \lambda_{2} / \log \lambda_{1}$ is irrational or $\mathscr{M}_{2}$ is of type $\mathrm{III}_{1}$, then the invariant $\left(\lambda_{3}, \mu_{3}\right)$ for $\alpha \otimes \beta$ is cohomologous to

$$
\left\{\begin{array}{l}
\lambda_{1}(n, g) \lambda_{2}(n, g) e^{i\left(\left(\tau_{1}(g)+\tau_{2}(g)\right) \nu_{3}\left(g^{-1} n g\right)-\tau_{1}(g) v_{1}\left(g^{-1} n g\right)-\tau_{2}(g) v_{2}\left(g^{-1} n g\right)\right)} ; \\
\overline{\mu_{1}}(m, n) \overline{\mu_{2}}(m, n) .
\end{array}\right.
$$

PROOF. (1) By identifying $L^{\infty}\left(\left[0,-\log \lambda_{3}\right) \times\{0\}\right)=L^{\infty}\left(\left[0,-\log \lambda_{3}\right)\right)$ with

$$
\left\{f \in L^{\infty}\left(\left(0,-\log \lambda_{1}\right) \times\left[0,-\log \lambda_{2}\right)\right): f\left(w_{1}-t, w_{2}+t\right)=f\left(w_{1}, w_{2}\right)\right\},
$$

we may regard $\lambda_{3}$ and $\mu_{3}$ in (2.12)-(2.13) as

$$
\left\{\begin{aligned}
\lambda_{3}(n, g)= & \lambda_{1}(n, g) \lambda_{2}(n, g) e^{i\left(\tau_{1}(g) k_{1}\left(g^{-1} n g\right) T_{1}+\tau_{2}(g) k_{2}\left(g^{-1} n g\right) T_{2}\right)} \\
& \times e^{-i w\left(k_{1}\left(g^{-1} n g\right)-k_{1}(n)\right) T_{1}} \\
\mu_{3}(m, n)= & \overline{\mu_{1}}(m, n) \overline{\mu_{2}}(m, n) e^{i w\left(v_{1}(m n)-v_{1}(m)-v_{1}(n)\right)} e^{i w\left(k_{1}(m n)-k_{1}(m)-k_{1}(n)\right) T_{1}}
\end{aligned}\right.
$$


for $w \in\left[0,-\log \lambda_{3}\right)$. Since $k_{1}(n) T_{1}+\nu_{1}(n)-\nu_{3}(n) \in T_{3} \mathbb{Z}$, we can consider a function $f(n)$ on $\left[0,-\log \lambda_{3}\right)$

$$
f(n)(w)=e^{i w\left(k_{1}(n) T_{1}+v_{1}(n)-v_{3}(n)\right)} .
$$

We perturb $\lambda_{3}$ and $\mu_{3}$ by $f(n)$. Then we have, by $\nu_{1}\left(g^{-1} n g\right)=\nu_{1}(n)$ and $\nu_{3}\left(g^{-1} n g\right)=$ $v_{3}(n)$,

$$
\begin{aligned}
& \lambda_{3}(n, g)\left(\tilde{\alpha}_{g} \otimes \tilde{\beta}_{g}\right)\left(f\left(g^{-1} n g\right)\right) f(n)^{*} \\
& \quad=\lambda_{3}(n, g) e^{i\left(w-\tau_{1}(g)-\tau_{2}(g)\right)\left(k_{1}\left(g^{-1} n g\right) T_{1}+v_{1}\left(g^{-1} n g\right)-v_{3}\left(g^{-1} n g\right)\right)} e^{-i w\left(k_{1}(n) T_{1}+v_{1}(n)-v_{3}(n)\right)} \\
& \quad=\lambda_{3}(n, g) e^{i w\left(k_{1}\left(g^{-1} n g\right)-k_{1}(n)\right) T_{1}} e^{-i\left(\tau_{1}(g)+\tau_{2}(g)\right)\left(k_{1}\left(g^{-1} n g\right) T_{1}+v_{1}\left(g^{-1} n g\right)-v_{3}\left(g^{-1} n g\right)\right)},
\end{aligned}
$$

since $\nu_{1}(n)+k_{1}(n) T_{1}=\nu_{2}(n)+k_{2}(n) T_{2}$ and (2.17),

$$
=\lambda_{1}(n, g) \lambda_{2}(n, g) e^{i\left(\left(\tau_{1}(g)+\tau_{2}(g)\right) \nu_{3}\left(g^{-1} n g\right)-\tau_{1}(g) \nu_{1}\left(g^{-1} n g\right)-\tau_{2}(g) \nu_{2}\left(g^{-1} n g\right)\right)},
$$

and

$$
\begin{aligned}
& \mu_{3}(m, n) f(m) f(n) f(m n)^{*} \\
& \quad=\mu_{3}(m, n) e^{i \omega\left(k_{1}(m) T_{1}+v_{1}(m)-v_{3}(m)+k_{1}(n) T_{1}+v_{1}(n)-v_{3}(n)-\left(k_{1}(m n) T_{1}+v_{1}(m n)-v_{3}(m n)\right)\right)} \\
& \quad=\overline{\mu_{1}}(m, n) \overline{\mu_{2}}(m, n) e^{i w\left(v_{3}(m n)-v_{3}(m)-v_{3}(n)\right)} .
\end{aligned}
$$

(2) Since $v(m n)=v(m)+v(n), k\left(g^{-1} n g\right)=k(n)$ and $k(m n)=k(m)+k(n)$, we have, by (2.15),

$$
\left\{\begin{array}{l}
\lambda_{3}(n, g)=\lambda_{1}(n, g) \lambda_{2}(n, g) e^{i \tau_{1}(g) k_{1}\left(g^{-1} n g\right) T_{1}} ; \\
\mu_{3}(n, g)=\overline{\mu_{1}}(m, n) \overline{\mu_{2}}(m, n) .
\end{array}\right.
$$

It is easy to show (use (2.9)) that

$$
\tau_{1}(g) k_{1}\left(g^{-1} n g\right) T_{1}=\left(\tau_{1}(g)+\tau_{2}(g)\right) \nu_{3}\left(g^{-1} n g\right)-\tau_{1}(g) \nu_{1}\left(g^{-1} n g\right)-\tau_{2}(g) \nu_{2}\left(g^{-1} n g\right) .
$$

Thus we obtain the conclusion of (2).

\section{Characteristic invariant for discrete crossed product}

Here we deal with characteristic invariant and modular invariant of the action induced up to a discrete crossed product and we give an example in which its invariants are computed explicitly.

Let $G$ and $H$ be discrete groups and $\alpha$ and $\beta$ be actions of $G$ and $H$ on an AFD factor $\mathscr{M}$ with $\alpha_{g} \beta_{h}=\beta_{h} \alpha_{g}$ for $g \in G$ and $h \in H$. The action $\beta$ is supposed to be an outer action of an amenable group $H$ in order that a crossed product $\mathscr{M} \rtimes_{\beta} H$ is an 
AFD factor. The action $\alpha$ of $G$ on $\mathscr{M}$ can be extended to an action (which is denoted by $\bar{\alpha}$ ) on the discrete crossed product $\mathscr{M} \rtimes_{\beta} H$ satisfying

$$
\bar{\alpha}_{g}\left(\pi_{\beta}(x)\right)=\pi_{\beta}\left(\alpha_{g}(x)\right), \quad \bar{\alpha}_{g}\left(\lambda_{\beta}(h)\right)=\lambda_{\beta}(h), \quad x \in \mathscr{M}, h \in H,
$$

where $\mathscr{M} \rtimes_{\beta} H$ is generated by $\left\{\pi_{\beta}(x), \lambda_{\beta}(h): x \in \mathscr{M}, h \in H\right\}$. In this section we compute the characteristic invariant and modular invariant for the action $\bar{\alpha}$. By perturbing an action $\alpha \times \beta_{(g, h)}=\alpha_{g} \beta_{h}$ by a cocycle, we may assume that it admits an $\alpha \times \beta$-invariant dominant weight $\varphi$ on $\mathscr{M}$ [20, Proposition 1.1]. We extend the actions $\alpha$ and $\beta$ to actions $\tilde{\alpha}$ and $\tilde{\beta}$ on $\mathscr{N}=\mathscr{M} \rtimes_{\sigma^{\varphi}} \mathbb{R}$. Since $\left(\mathscr{M} \rtimes_{\beta} H\right) \rtimes_{\sigma^{\dot{\varphi}}} \mathbb{R}$ is canonically isomorphic to $\mathscr{N} \rtimes_{\tilde{\beta}} H$, where $\tilde{\varphi}$ a dual weight of $\varphi$ [18], we may regard the action $\tilde{\bar{\alpha}}$ as

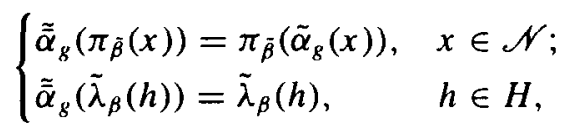

where $\left\{\pi_{\tilde{\beta}}(x), \tilde{\lambda}_{\beta}(h): x \in \mathscr{N}, h \in H\right\}$ generates $\mathscr{N} \rtimes_{\tilde{\beta}} H$. The action $\tilde{\tilde{\alpha}}$ is denoted by the same symbol $\bar{\alpha}$. Let $N_{\beta}$ be a normal subgroup of $H$ defined by $\tilde{\beta}^{-1}(\operatorname{Int}(\mathscr{N}))$ and $(\lambda, \mu)$ and $c(n, t)$ be the characteristic invariant and modular invariant of $\alpha \times \beta$. A twisted crossed product $\mathscr{C} \rtimes_{i d, \mu_{\beta}} N_{\beta}$ of the centre $\mathscr{C}=Z(\mathscr{N})$ by trivial action plav's a crucial role in the description of invariants for $\bar{\alpha}([18])$, where $\mu_{\beta}$ is a restriction of $\mu$ on $N_{\beta}$. The invariants $\lambda$ and $c(n, t)$ give actions $\gamma$ of $H$ and $F$ of $\mathbb{R}$ on $\mathscr{C} \rtimes_{i d, \mu_{\beta}} N_{\beta}$ for an element $\sum_{l \in N_{\beta}} d_{l} z_{l} \in \mathscr{C} \rtimes_{i d, \mu_{\beta}} N_{\beta}$ as follows

$$
\left\{\begin{array}{l}
\gamma_{k}\left(\sum_{l \in N_{\beta}} d_{l} z_{l}\right)=\sum_{l \in N_{\beta}} \lambda(l, k) \tilde{\beta}_{k}\left(d_{k l k^{-1}}\right) z_{l} ; \\
F_{t}\left(\sum_{l \in N_{\beta}} d_{l} z_{l}\right)=\sum_{l \in N_{\beta}} \theta_{t}\left(d_{l}\right) c(l, t) z_{l},
\end{array}\right.
$$

where $d_{l} \in \mathscr{C}, z_{l_{3}} z_{l_{2}}=\mu_{\beta}\left(l_{1}, l_{2}\right) z_{l_{1} l_{2}}$ and $\theta_{t}$ is the flow on $\mathscr{C}$. Moreover, for $g \in G$, we define an action $\rho$ of $G$ by

$$
\rho_{g}\left(\sum_{l \in N_{\beta}} d_{l} z_{l}\right)=\sum_{l \in N_{g}} \lambda(l, g) \tilde{\alpha}_{g}\left(d_{l}\right) z_{l} .
$$

We set a normal subgroup $N_{\alpha \times \beta}=(\widetilde{\alpha \times \beta})^{-1}(\operatorname{Int}(\mathscr{N}))$ of $G \times H$ and $a_{n, h}(k) \epsilon$ $\mathscr{C} \rtimes_{i d, \mu_{\beta}} N_{\beta}$ : for $(n, h) \in N_{\alpha \times \beta}$, and $k \in H$,

$$
a_{n, h}(k)=\lambda\left(\left(n, k h k^{-1}\right), k\right) \mu\left((n, h),\left(e, h^{-1} k h k^{-1}\right)\right)^{*} z_{h^{-1} k h k^{-1}} .
$$


LEMMA 3.1. For $(n, h) \in N_{\alpha \times \beta}$, the $a_{n, h}$ is $\gamma$-cocycle in $\mathscr{C} \rtimes_{i d, \mu_{\beta}} N_{\beta}$. Namely, $a_{n, h}(k) \gamma_{k}\left(a_{n, h}(l)\right)=a_{n, h}(k l)$. Moreover, $a_{n, h}$ and $a_{n, l}$ are cohomologous with $\mu\left((n, h),\left(e, h^{-1} l\right)\right)^{*} z_{h^{-1} l}$, for $(n, h),(n, l) \in N_{\alpha \times \beta}$, namely

$$
a_{n, l}(k)=\left(\mu\left((n, h),\left(e, h^{-1} l\right)\right)^{*} z_{h^{-1} l}\right)^{*} a_{n, h}(k) \gamma_{k}\left(\mu\left((n, h),\left(e, h^{-1} l\right)\right)^{*} z_{h^{-1} l}\right)
$$

for $k \in H$.

PROOF. We set $a_{n, h}^{\prime}(k)=\gamma_{k}^{\prime}\left(u(n, h) \tilde{\lambda}_{\beta}(h)^{*}\right)\left(u(n, h) \tilde{\lambda}_{\beta}(h)^{*}\right)^{*}$, where

$$
\gamma_{l}^{\prime}=\left.\operatorname{Ad} \tilde{\lambda}_{\beta}(l)\right|_{\mathcal{N} \rtimes_{\bar{\beta}} H} \quad \text { and } \quad \tilde{\alpha}_{n} \tilde{\beta}_{h}=\operatorname{Ad} u(n, h) .
$$

Since $\left.\operatorname{Ad} u(n, h) \tilde{\lambda}_{\beta}(h)^{*}\right|_{\mathcal{N}}=\tilde{\alpha}_{n}$ and $\tilde{\beta}_{k}$ commutes with $\tilde{\alpha}$, the $a_{n, h}^{\prime}(k)$ is an element of $\mathscr{N}^{\prime} \cap\left(\mathscr{N} \rtimes_{\tilde{\beta}} H\right)$. We compute, using $k h^{-1} k^{-1} h \in N_{\beta}$,

$$
\begin{aligned}
a_{n, h}^{\prime}(k)= & \tilde{\beta}_{k}(u(n, h)) \tilde{\lambda}_{\beta}\left(k h^{-1} k^{-1} h\right) u(n, h)^{*} \\
= & \lambda\left(\left(n, k h k^{-1}\right), k\right) u\left(n, k h k^{-1}\right) \tilde{\beta}_{h^{-1} k h k^{-1}}^{-1}\left(u(n, h)^{*}\right) \tilde{\lambda}_{\beta}\left(h^{-1} k h k^{-1}\right)^{*} \\
= & \lambda\left(\left(n, k h k^{-1}\right), k\right) u\left(n, k h k^{-1}\right) \\
& \times u\left(e, h^{-1} k h k^{-1}\right)^{*} u(n, h)^{*} u\left(e, h^{-1} k h k^{-1}\right) \tilde{\lambda}_{\beta}\left(h^{-1} k h k^{-1}\right)^{*} \\
= & \lambda\left(\left(n, k h k^{-1}\right), k\right) u\left(n, k h k^{-1}\right) \mu\left((n, h),\left(e, h^{-1} k h k^{-1}\right)\right)^{*} u\left(n, k h k^{-1}\right)^{*} \\
& \times u\left(e, h^{-1} k h k^{-1}\right) \tilde{\lambda}_{\beta}\left(h^{-1} k h k^{-1}\right)^{*} \\
= & \lambda\left(\left(n, k h k^{-1}\right), k\right) \mu\left((n, h),\left(e, h^{-1} k h k^{-1}\right)\right)^{*} \\
& \times u\left(e, h^{-1} k h k^{-1}\right) \tilde{\lambda}_{\beta}\left(h^{-1} k h k^{-1}\right)^{*} .
\end{aligned}
$$

By the anti-isomorphism $\Pi$ in [18, Lemma 2.4], we have $\Pi\left(a_{n, h}(k)\right)=a_{n, h}^{\prime}(k)$. It follows from the definition of $a_{n, h}^{\prime}(k)$ that $a_{n, h}^{\prime}(k)$ satisfies $\gamma_{k}^{\prime}\left(a_{n, h}^{\prime}(l)\right) a_{n, h}^{\prime}(k)=a_{n, h}^{\prime}(k l)$. Therefore, $a_{n, h}(k)$ satisfies $a_{n, h}(k) \gamma_{k}\left(a_{n, h}(l)\right)=a_{n, h}(k l)$. We choose another unitary $u(n, l)$ satisfying $\widetilde{\alpha \times \beta}_{(n, l)}=\operatorname{Ad} u(n, l)$ for $(n, l) \in N_{\alpha \times \beta}$. Then we have

$$
\operatorname{Ad} u(n, h)^{*} \operatorname{Ad} u(n, l)=\widetilde{\alpha \times \beta_{(n, h)}^{-1}} \widetilde{\alpha \times \beta_{(n, l)}}=\tilde{\beta}_{h^{-1} l}=\left.\operatorname{Ad} \tilde{\lambda}_{\beta}\left(h^{-1} l\right)\right|_{\mathcal{N}} .
$$

Therefore, there is $d \in \mathscr{N}^{\prime} \cap\left(\mathscr{X} \rtimes_{\bar{\beta}} H\right)$ such that $u(n, l)=d \cdot u(n, h) \tilde{\lambda}_{\beta}\left(h^{-1} l\right)$ and we have

$$
\begin{aligned}
d & =u(n, h)^{*} u(n, l) \tilde{\lambda}_{\beta}\left(h^{-1} l\right)^{*}=u(n, h)^{*} u\left((n, h) \cdot\left(e, h^{-1} l\right)\right) \tilde{\lambda}_{\beta}\left(h^{-1} l\right)^{*} \\
& =u(n, h)^{*} \mu\left((n, h),\left(e, h^{-1} l\right)\right)^{*} u(n, h) u\left(e, h^{-1} l\right) \tilde{\lambda}_{\beta}\left(h^{-1} l\right) \\
& =\mu\left((n, h),\left(e, h^{-1} l\right)\right)^{*} u\left(e, h^{-1} l\right) \tilde{\lambda}_{\beta}\left(h^{-1} l\right) .
\end{aligned}
$$

Therefore, $\Pi\left(\mu\left((n, h),\left(e, h^{-1} l\right)\right)^{*} z_{h^{-1} l}\right)=d$. Since

$$
\begin{aligned}
a_{n, l}^{\prime}(k) & =\gamma_{k}(d) \gamma_{k}\left(u(n, h) \tilde{\lambda}_{\beta}\left(k^{-1} l\right) \tilde{\lambda}_{\beta}(l)^{*}\right) \tilde{\lambda}_{\beta}(l) \tilde{\lambda}_{\beta}\left(k^{-1} l\right)^{*} u(n, h)^{*} d^{*} \\
& =\gamma_{k}(d) a_{n, h}^{\prime}(k) d^{*},
\end{aligned}
$$


we conclude

$$
a_{n, l}(k)=\left(\mu\left((n, h),\left(e, h^{-1} l\right)\right)^{*} z_{h^{-1} l}\right)^{*} a_{n, h}(k) \gamma_{k}\left(\left(\mu\left((n, h),\left(e, h^{-1} l\right)\right)^{*} z_{h^{-1 l}}\right) .\right.
$$

REMARK. If the group $H$ is abelian, the $\gamma$-cocycle $a_{n, h}(k)$ is just $\lambda((n, h), k)$. It follows from (2) in the definition for $\lambda$ that $\lambda((n, h), \cdot)$ is $\gamma$-cocycle. By making use of the definition (1)-(4) for the characteristic invariant, we can prove Lemma 3.1 in an algebraic way, but its proof is rather complicated.

Next we shall show that the characteristic invariant and modular invariant for $\bar{\alpha}$ can be expressed as the operators in $\mathscr{C} \rtimes_{i d . \mu_{\beta}} N_{\beta}$ by making use of the anti-isomorphism $\Pi$ in [18, Lemma 2.4].

THEOREM 3.2. Let $N_{\hat{\alpha}}$ be a normal subgroup $\bar{\alpha}^{-1}\left(\operatorname{Int}\left(\mathscr{N} \rtimes_{\tilde{\beta}} H\right)\right)$ of $G$ and let $\left[a_{n, h(n)}\right]$ denote the class of $a_{n, h(n)}(k)$ in $\mathrm{H}_{\gamma}^{1}\left(H, \mathscr{C} \rtimes_{i d, \mu_{\beta}} N_{\beta}\right)$.

(1) The group $N_{\bar{\alpha}}$ is

$$
\left\{n \in G:(n, h(n)) \in N_{\alpha \times \beta} \text { and }\left[a_{n, h(n)}\right]=0 \text {, for some } h(n) \in H\right\} \text {. }
$$

(2) The characteristic invariants $(\bar{\lambda}, \bar{\mu})$ in $\left(\mathscr{C} \rtimes_{i d, \mu_{\beta}} N_{\beta}\right)^{\gamma}$ for $\bar{\alpha}$ are given by

$$
\left\{\begin{aligned}
\bar{\lambda}(n, g)= & \lambda\left(\left(n, h\left(g^{-1} n g\right)\right), g\right) \\
& \times \mu\left((n, h(n)),\left(e, h(n)^{-1} h\left(g^{-1} n g\right)\right)\right)^{*} \\
& \times z_{h(n)^{-1} h\left(g^{-1} n g\right)} \rho_{g}\left(b\left(g^{-1} n g\right)\right) b(n)^{*} ; \\
\bar{\mu}(n, m)= & \lambda\left(\left(n, h(m)^{-1} h(n) h(m)\right), h(m)^{-1}\right) \\
& \times \mu\left((m, h(m)),\left(n, h(m)^{-1} h(n) h(m)\right)\right) \\
& \times \mu\left((m n, h(m n)),\left(e, h(m n)^{-1} h(n) h(m)\right)\right)^{*} \\
& \times z_{h(m n)^{-1} h(n) h(m)} \gamma_{h(m)}^{-1}(b(n)) b(m) b(m n)^{*}
\end{aligned}\right.
$$

for $(n, h(n)),(m, h(m)) \in N_{\alpha \times \beta}, g \in G$.

The modular invariant $\bar{c}(n)$ is given by

$$
\bar{c}(n)(t)=c(n, h(n))(t) F_{t}(b(n)) b(n)^{*},
$$

where $a_{n, h(n)}(k)=b(n) \gamma_{k}\left(b(n)^{*}\right)$ for some $b(n) \in \mathscr{C} \rtimes_{i d, \mu_{\beta}} N_{\beta}$ and $\rho, \gamma$ and $F$ are given in (3.3) and (3.4).

PROOF. (1) We note, firstly, that the cohomology class of $a_{n, h}$ is independent of the choice of $h(n)$ by Lemma 3.1. Take $n \in N_{\bar{\alpha}}$ and choose a unitary $U_{n} \in \mathscr{N} \rtimes_{\bar{\beta}} H$ such that $\bar{\alpha}_{n}=\operatorname{Ad} U_{n}$ on $\mathscr{N} \rtimes_{\bar{\beta}} H$. Since $U_{n}$ is of the form

$$
\sum_{h \in H} v_{h} \tilde{\lambda}_{\beta}(h)
$$


where $v_{h} \in \mathscr{N}$, it follows from $U_{n} x=\bar{\alpha}_{n}(x) U_{n}, x \in \mathscr{N} \rtimes_{\tilde{\beta}} H$ that

$$
\sum_{h \in H} v_{h} \tilde{\beta}_{h}(x) \tilde{\lambda}_{\beta}(h)=\tilde{\alpha}_{n}(x) \sum_{h \in H} v_{h} \tilde{\lambda}_{\beta}(h), \quad \text { for } x \in \mathscr{N}
$$

Hence we have $v_{h^{-1}} x=\tilde{\alpha}_{n} \tilde{\beta}_{h}(x) v_{h^{-1}}$ for $h \in H$. By [18, Lemma 2.3], if $\tilde{\alpha}_{n} \tilde{\beta}_{h}$ is not inner, then $v_{h}$ must be zero. Hence there is $h(n) \in H$ such that $\tilde{\alpha}_{n} \tilde{\beta}_{h(n)}$ is an inner automorphism of $\mathscr{N}$. We choose unitary $u(n, h(n)) \in \mathscr{U}(\mathscr{N})$ such that $\tilde{\alpha}_{n} \tilde{\beta}_{h(n)}=\operatorname{Ad} u(n, h(n))$. We compute, for $x \in \mathscr{N}$,

$$
\operatorname{Ad} u(n, h(n)) \tilde{\lambda}_{\beta}(h(n))^{*}(x)=\tilde{\alpha}_{n} \tilde{\beta}_{h(n)} \tilde{\beta}_{h(n)}^{-1}(x)=\tilde{\alpha}_{n}(x)=\operatorname{Ad} U_{n}(x) .
$$

We set $b^{\prime}(n)^{*}=u(n, h(n)) \tilde{\lambda}_{\beta}(h(n))^{*} U_{n}^{*} \in \mathscr{N}^{\prime} \cap\left(\mathscr{N} \rtimes_{\tilde{\beta}} H\right)$. Since the extended automorphism $\bar{\alpha}_{n}$ satisfies $\bar{\alpha}_{n}\left(\tilde{\lambda}_{\beta}\left(k^{-1}\right)\right)=\tilde{\lambda}_{\beta}\left(k^{-1}\right)$ for $k \in H$, we have

$$
\begin{aligned}
\tilde{\lambda}_{\beta}\left(k^{-1}\right) & =\bar{\alpha}_{n}\left(\tilde{\lambda}_{\beta}\left(k^{-1}\right)\right)=U_{n} \tilde{\lambda}_{\beta}\left(k^{-1}\right) U_{n}^{*} \\
& =b^{\prime}(n) u(n, h(n)) \tilde{\lambda}_{\beta}(h(n))^{*} \tilde{\lambda}_{\beta}\left(k^{-1}\right) \tilde{\lambda}_{\beta}(h(n)) u(n, h(n))^{*} b^{\prime}(n)^{*} .
\end{aligned}
$$

This implies that

$$
\gamma_{k}\left(b^{\prime}(n)^{*}\right) b^{\prime}(n)=\gamma_{k}\left(u(n, h(n)) \tilde{\lambda}_{\beta}(h(n))^{*}\right)\left(u(n, h(n)) \tilde{\lambda}_{\beta}(h(n))^{*}\right)^{*}=a_{n, h(n)}^{\prime}(k)
$$

and we have

$$
b(n) \gamma_{k}\left(b(n)^{*}\right)=\Pi\left(b^{\prime}(n)\right) \gamma_{k}\left(\Pi\left(b^{\prime}(n)^{*}\right)\right)=\Pi\left(\gamma_{k}\left(b^{\prime}(n)^{*}\right) b^{\prime}(n)\right)=a_{n, h(n)} .
$$

Conversely, suppose that there is $b(n) \in \mathscr{C} \rtimes_{i d, \mu_{\beta}} N_{\beta}$ such that $b(n) \gamma_{k}(b(n))^{*}=a_{n, h(n)}$ for some $(n, h(n)) \in N_{\alpha \times \beta}$. We set

$$
U_{n}=b^{\prime}(n) u(n, h(n)) \tilde{\lambda}_{\beta}(h(n))^{*},
$$

where $b^{\prime}(n)=\Pi^{-1}(b(n))$. Then we have for $x \in \mathscr{N}, k \in H$,

$$
\begin{aligned}
& \operatorname{Ad} U_{n}(x)=\operatorname{Ad} b^{\prime}(n) \tilde{\alpha}_{n}(x)=\tilde{\alpha}_{n}(x) ; \\
& \begin{aligned}
\operatorname{Ad} U_{n} & \left(\tilde{\lambda}_{\beta}\left(k^{-1}\right)\right) \\
& =b^{\prime}(n) u(n, h(n)) \tilde{\lambda}_{\beta}(h(n))^{*} \tilde{\lambda}_{\beta}\left(k^{-1}\right) \tilde{\lambda}_{\beta}(h(n)) u(n, h(n))^{*} b^{\prime}(n)^{*} \\
& =\tilde{\lambda}\left(k^{-1}\right) \gamma_{k}\left(b^{\prime}(n)\right) \gamma_{k}\left(u(n, h(n)) \tilde{\lambda}_{\beta}(h(n))^{*}\right)\left(u(n, h(n)) \tilde{\lambda}_{\beta}(h(n))^{*}\right)^{*} b^{\prime}(n)^{*} \\
& =\tilde{\lambda}_{\beta}\left(k^{-1}\right) \gamma_{k}\left(b^{\prime}(n)\right) a_{n, k}^{\prime}(k) b^{\prime}(n)^{*}=\tilde{\lambda}_{\beta}\left(k^{-1}\right) .
\end{aligned}
\end{aligned}
$$

Hence the automorphism $\bar{\alpha}_{n}$ on $\mathscr{N} \rtimes_{\bar{\beta}} H$ is inner with the unitary $U_{n}$ in $\mathscr{N} \rtimes_{\bar{\beta}} H$. Thus we have proved the statement (1). 
(2) Let $U_{n}$ be as in (3.8). We compute

$$
\begin{aligned}
\bar{\alpha}_{g}\left(U_{g^{-1} n g}\right) U_{n}^{*}= & \bar{\alpha}_{g}\left(b^{\prime}\left(g^{-1} n g\right) u\left(g^{-1} n g, h\left(g^{-1} n g\right)\right) \tilde{\lambda}_{\beta}\left(h\left(g^{-1} n g\right)\right)^{*}\right) \\
& \times \tilde{\lambda}_{\beta}(h(n)) u(n, h(n))^{*} b^{\prime}(n)^{*} \\
= & b^{\prime}(n)^{*} \bar{\alpha}_{g}\left(b^{\prime}\left(g^{-1} n g\right)\right) \lambda\left(\left(n, h\left(g^{-1} n g\right)\right), g\right) u\left(n, h\left(g^{-1} n g\right)\right) \\
& \times \tilde{\lambda}_{\beta}\left(h\left(g^{-1} n g\right)^{-1} h(n)\right) u(n, h(n))^{*} \\
= & b^{\prime}(n)^{*} \bar{\alpha}_{g}\left(b^{\prime}\left(g^{-1} n g\right)\right) \lambda\left(\left(n, h\left(g^{-1} n g\right)\right), g\right) u\left(n, h\left(g^{-1} n g\right)\right) \\
& \times \tilde{\beta}_{h\left(g^{-1} n g\right)^{-1} h(n)}\left(u(n, h(n))^{*}\right) \tilde{\lambda}_{\beta}\left(h(n)^{-1} h\left(g^{-1} n g\right)\right)^{*} \\
= & b^{\prime}(n)^{*} \bar{\alpha}_{g}\left(b^{\prime}\left(g^{-1} n g\right)\right) \lambda\left(\left(n, h\left(g^{-1} n g\right)\right), g\right) u\left(n, h\left(g^{-1} n g\right)\right) \\
& \times u\left(e, h(n)^{-1} h\left(g^{-1} n g\right)\right)^{*} u(n, h(n))^{*} u\left(e, h(n)^{-1} h\left(g^{-1} n g\right)\right) \\
& \times \tilde{\lambda}_{\beta}\left(h(n)^{-1} h\left(g^{-1} n g\right)\right)^{*} \\
= & b^{\prime}(n)^{*} \bar{\alpha}_{g}\left(b^{\prime}\left(g^{-1} n g\right)\right) \lambda\left(\left(n, h\left(g^{-1} n g\right)\right), g\right) \\
& \times \mu\left((n, h(n)),\left(e, h(n)^{-1} h\left(g^{-1} n g\right)\right)\right)^{*} \\
& \times u\left(e, h(n)^{-1} h\left(g^{-1} n g\right)\right) \tilde{\lambda}_{\beta}\left(h(n)^{-1} h\left(g^{-1} n g\right)\right)^{*} .
\end{aligned}
$$

Then the characteristic invariant $\bar{\lambda}$ for $\bar{\alpha}$ is of the form

$$
\begin{aligned}
\bar{\lambda}(n, g)= & \lambda\left(\left(n, h\left(g^{-1} n g\right)\right), g\right) \mu\left((n, h(n)),\left(e, h(n)^{-1} h\left(g^{-1} n g\right)\right)\right)^{*} \\
& \times z_{h(n)^{-1} h\left(g^{-1} n g\right)} \rho_{g}\left(b\left(g^{-1} n g\right)\right) b(n)^{*} .
\end{aligned}
$$

We compute

$$
\begin{aligned}
U_{m} U_{n} U_{m n}^{*}= & b^{\prime}(m) u(m, h(m)) \tilde{\lambda}_{\beta}(h(m))^{*} b^{\prime}(n) u(n, h(n)) \tilde{\lambda}_{\beta}(h(n))^{*} \\
& \times\left(b^{\prime}(m n) u(m n, h(m n)) \tilde{\lambda}_{\beta}(h(m n))^{*}\right)^{*} \\
= & b^{\prime}(m n)^{*} b^{\prime}(m) \gamma_{h(m)}^{-1}\left(b^{\prime}(n)\right) u(m, h(m)) \tilde{\lambda}_{\beta}(h(m))^{*} u(n, h(n)) \\
& \times \tilde{\lambda}_{\beta}(h(n))^{*} \tilde{\lambda}_{\beta}(h(m n)) u(m n, h(m n))^{*} \\
= & b^{\prime}(m n)^{*} b^{\prime}(m) \gamma_{h(m)}^{\prime-1}\left(b^{\prime}(n)\right) u(m, h(m)) \lambda\left(\left(n, h(m)^{-1} h(n) h(m)\right), h(m)^{-1}\right) \\
& \times u\left(n, h(m)^{-1} h(n) h(m)\right) \tilde{\lambda}_{\beta}\left(h(m)^{-1} h(n)^{-1} h(m n)\right) u(m n, h(m n))^{*} \\
= & b^{\prime}(m n)^{*} b^{\prime}(m) \gamma_{h(m)}^{\prime-1}(b(n)) \lambda\left(\left(n, h(m)^{-1} h(n) h(m)\right), h(m)^{-1}\right) \\
& \times u(m, h(m)) u\left(n, h(m)^{-1} h(n) h(m)\right) u\left(e, h(m n)^{-1} h(n) h(m)\right)^{*} \\
& \times u(m n, h(m n))^{*} u\left(e, h(m n)^{-1} h(n) h(m)\right) \tilde{\lambda}_{\beta}\left(h(m n)^{-1} h(n) h(m)\right)^{*} \\
= & b^{\prime}(m n)^{*} b^{\prime}(m) \gamma_{h(m)}^{-1}\left(b^{\prime}(n)\right) \lambda\left(\left(n, h(m)^{-1} h(n) h(m)\right), h(m)^{-1}\right) \\
& \times \mu\left((m, h(m)),\left(n, h(m)^{-1} h(n) h(m)\right)\right) \\
& \times \mu\left((m n, h(m n)),\left(e, h(m n)^{-1} h(n) h(m)\right)\right)^{*} \\
& \times u\left(e, h(m n)^{-1} h(n) h(m)\right) \tilde{\lambda}_{\beta}\left(h(m n)^{-1} h(n) h(m)\right)^{*} .
\end{aligned}
$$


Then we obtain

$$
\begin{aligned}
\bar{\mu}(n, m)= & \lambda\left(\left(n, h(m)^{-1} h(n) h(m)\right), h(m)^{-1}\right) \mu\left((m, h(m)),\left(n, h(m)^{-1} h(n) h(m)\right)\right. \\
& \times \mu\left((m n, h(m n)),\left(e, h(m n)^{-1} h(n) h(m)\right)\right)^{*} z_{h(m n)^{-1} h(n) h(m)} \\
& \times \gamma_{h(m)}^{-1}(b(n)) b(m) b(m n)^{*}
\end{aligned}
$$

Finally, we compute

$$
\begin{aligned}
\tilde{\theta}_{t}\left(U_{n}\right) U_{n}^{*} & =b^{\prime}(n)^{*} \tilde{\theta}_{t}\left(b^{\prime}(n)\right) c(n, h(n))(t) u(n, h(n)) \tilde{\lambda}_{\beta}(h(n))^{*} \tilde{\lambda}_{\beta}(h(n)) u(n, h(n))^{*} \\
& =b^{\prime}(n)^{*} \tilde{\theta}_{t}\left(b^{\prime}(n)\right) c(n, h(n))(t)
\end{aligned}
$$

where $\tilde{\theta}_{t}$ is a dual action on $\mathscr{N} \rtimes_{\tilde{\beta}} H$ for the modular automorphism $\sigma^{\tilde{\varphi}}$. Then we obtain $\bar{c}(n, t)=c(n, h(n))(t) F_{t}(b(n)) b(n)^{*}$.

From now on, we assume that the group $H$ is abelian and the factor $\mathscr{M}$ is of type III $_{\lambda}(0<\lambda \leqq 1)$. We shall give a form of $b(n)$ in Theorem 3.2 and the invariants $\bar{\lambda}, \bar{\mu}, \bar{c}$ explicitly. If $\mathscr{M}$ is of type III $_{\lambda}(0<\lambda<1)$, we may assume that the invariants $(\lambda, \mu)$ and $\nu$ for the action $\alpha \times \beta$ of $G \times H$ are as in Lemma 2.2. Since the $\gamma$ cocycle $a_{n, h}(k)$ is $\lambda((n, h), k)$, it follows from (2) in the definition for $\lambda$ that a map $k \in H \rightarrow \lambda((n, h), k) \in \mathbb{T}$ is a character of $H$. Therefore, we define $\Phi(n, h) \in \widehat{H}$ by

$$
\langle k, \Phi(n, h)\rangle=\lambda((n, h), k)
$$

for $k \in H$, where $\widehat{H}$ is a dual group of $H$. For $p \in \mathbb{Z}$, the map $l \in H \rightarrow e^{i \tau(l) p T} \in \mathbb{T}$ is also a character of $H$, where $T=-2 \pi / \log \lambda$ and we define $\Psi(p) \in \widehat{H}$ by

$$
\langle l, \Psi(p)\rangle=e^{i \tau(l) p T}
$$

for $l \in H$. Then the map $\Psi: p \in \mathbb{Z} \rightarrow \Psi(p) \in \widehat{H}$ is a homomorphism. By (2.7), we have

$$
\begin{aligned}
\lambda((m, h), k) \lambda((n, l), k) & =\lambda((m n, h l), k) \tilde{\beta}_{k}(\mu((m, h),(n, l))) \mu((m, h),(n, l))^{*} \\
& =\lambda((m n, h l), k) e^{i \tau(k)(v(m, h)+v(n, l)-v(m n, h l))}
\end{aligned}
$$

which implies that

$$
\Phi(m, h)+\Phi(n, l)=\Phi(m n, h l)+\Psi(v(m, h)+v(n, l)-v(m n, h l)) .
$$

Proposition 3.3. With notation as above, if $\mathscr{M}$ is of type $\mathrm{III}_{\lambda}(0<\lambda<1)$ (respectively $\mathrm{III}_{1}$ ), we have the following statements. 
(1) The $\gamma$-cocycle $a_{n, h}$, for $(n, h) \in N_{\alpha \times \beta}$ is coboundary with $b(n) \in \mathscr{C} \rtimes_{i d, \mu_{\beta}} N_{\beta}$, namely $a_{n, k}(k)=b(n) \gamma_{k}\left(b(n)^{*}\right)$ if and only if there is $l \in N_{\beta}$ such that

$$
\Psi(p)=\Phi(n, h)+\Phi(e, l), \quad \text { (respectively } \Phi(n, h)+\Phi(e, l)=e)
$$

for some $p \in \mathbb{Z}$. Moreover, we can choose $h(n) \in H$ and $p(n) \in \mathbb{Z}$ such that $(n, h(n)) \in N_{\alpha \times \beta}$ and $\Psi(p(n))=\Phi(n, h(n))$ (respectively $\left.\Phi(n, h(n))=e\right)$ and $b(n)$ can be chosen to be of the form $b(n)=e^{i w p(n) T} \in \mathscr{C} \rtimes_{i d, \mu_{\beta}} N_{\beta}$, (respectively $b(n)=1$ ), where $w \in[0,-\log \lambda)$.

(2) The invariants $\bar{\lambda}, \bar{\mu}$ and $\bar{c}$ are as follows

$$
\begin{aligned}
& \bar{\lambda}(n, g)=\lambda\left(\left(n, h\left(g^{-1} n g\right)\right), g\right) \mu\left((n, h(n)),\left(e, h(n)^{-1} h\left(g^{-1} n g\right)\right)\right)^{*} \\
& \times z_{h(n)^{-1} h\left(g^{-1} n g\right)} e^{i \omega\left(p\left(g^{-1} n g\right)-p(n)\right) T} e^{-i \tau(g) p\left(g^{-1} n g\right) T} ; \\
& \bar{\mu}(m, n)=\mu((m, h(m)),(n, h(n))) \lambda\left((n, h(n)), h(m)^{-1}\right) \\
& \times \mu\left((m n, h(m n)),\left(e, h(m n)^{-1} h(n) h(m)\right)^{*} z_{h(m n)^{-1} h(n) h(m)}\right. \\
& \times e^{i w(p(m)+p(n)-p(m n)) T} e^{-i \tau\left(h(m)^{-1}\right) p(n) T} ; \\
& \bar{c}(n)(t)=c(n, h(n))(t) e^{-i t p(n) T} . \\
& \left(\begin{array}{rl}
\text { respectively } & \\
\bar{\lambda}(n, g)= & \lambda\left(\left(n, h\left(g^{-1} n g\right)\right), g\right) \mu\left((n, h(n)),\left(e, h(n)^{-1} h\left(g^{-1} n g\right)\right)\right)^{*} \\
& \times z_{h(n)^{-1} h\left(g^{-1} n g\right)} ; \\
\bar{\mu}(m, n)= & \mu((m, h(m)),(n, h(n))) \lambda\left((n, h(n)), h(m)^{-1}\right) \\
& \times \mu\left((m n, h(m n)),\left(e, h(m n)^{-1} h(n) h(m)\right)^{*}\right. \\
& \times z_{h(m n)^{-1} h(n) h(m)} ; \\
\bar{c}(n)(t)= & c(n, h(n))(t) .
\end{array}\right)
\end{aligned}
$$

PROOF. (1) Suppose that there is $b(n) \in \mathscr{C} \rtimes_{i d, \mu_{\beta}} N_{\beta}$ with

$$
\langle k, \Phi(n, h)\rangle=b(n) \gamma_{k}\left(b(n)^{*}\right) .
$$

Since $b(n)=\sum_{l \in N_{\beta}} d_{l} z_{l}$ for $d_{l} \in \mathscr{C}$, we compute

$$
\left\{\begin{array}{l}
\gamma_{k}(b(n))=\sum_{l \in N_{\beta}} \tilde{\beta}_{k}\left(d_{l}\right) \lambda((e, l), k) z_{l} \\
\langle k,-\Phi(n, h)\rangle b(n)=\sum_{l \in N_{\beta}}\langle k,-\Phi(n, h)\rangle d_{l} z_{l} .
\end{array}\right.
$$

By the comparison of coefficients, we have

$$
\tilde{\beta}_{k}\left(d_{l}\right)=\langle k,-\Phi(n, h)-\Phi(e, l)\rangle d_{l}
$$


for all $l \in N_{\beta}$. Since $\mathscr{C}$ is isomorphic to $L^{\infty}([0,-\log \lambda))$, we have

$$
\tilde{\beta}_{k}\left(d_{l}\right)=\sum_{p \in \boldsymbol{Z}} d_{l, p} e^{i(w-\tau(k)) p} T
$$

where $d_{l}=\sum_{p \in \mathbb{I}} d_{l, p} e^{i \omega p T}$ (Fourier expansion of $d_{l}$ ). Then we obtain, again by the comparison of coefficients,

$$
d_{l, p} e^{-i \tau(k) p T}=\langle k,-\Phi(n, h)-\Phi(e, l)\rangle d_{l, p}
$$

for all $p \in \mathbb{Z}$. This implies that $\Psi(p)=\Phi(n, h)+\Phi(e, l)$ for some $p \in \mathbb{Z}$ and $l \in N_{\beta}$. Conversely, we suppose that for $n \in N_{\beta}$, there are $p \in \mathbb{Z}, l \in N_{\beta}$ and $h \in H$ with $(n, h) \in N_{\alpha \times \beta}$ such that $\Psi(p)=\Phi(n, h)+\Phi(e, l)$. We set

$$
b(n)=e^{i w p T} z_{l} \in \mathscr{C} \rtimes_{i d, \mu_{\beta}} N_{\beta},
$$

and compute

$$
\begin{aligned}
b(n) \gamma_{k}\left(b(n)^{*}\right) & =e^{i w p T} z_{l} z_{l}^{*} \overline{\lambda((e, l), k)} e^{-i(w-\tau(k)) p T}=e^{i \tau(k) p T} \overline{\lambda((e, l), k)} \\
& =\langle k, \Psi(p)-\Phi(e, l)\rangle=\langle k, \Phi(n, h)\rangle=\lambda((n, h), k)=a_{n, h}(k) .
\end{aligned}
$$

By (3.9), we set $p(n) \in \mathbb{Z}$ and $h(n) \in N_{\beta}$

$$
\left\{\begin{array}{l}
p(n)=p+v(n, h l)-v(n, h)-v(e, l) ; \\
h(n)=l h,
\end{array}\right.
$$

which satisfies $\Psi(p(n))=\Phi(n, h(n))$. Then we may take $b(n)=e^{i w p(n) T}$. Making use of $b(n),(3.6)$ and (3.7), we conclude that the statement (2) holds. Even when $\mathscr{M}$ is of type $\mathrm{II}_{1}$, we can prove the statement using the same argument.

\section{References}

[1] A. Connes, 'Une classification des facteurs de type III', Ann. Sci. École Norm. Sup. 6 (1973), 133-252.

[2] _ 'Almost periodic states and factors of type III', J. Funct. Anal. 16 (1974), 415-445.

[3] - 'Outer conjugacy classes of automorphisms of factors', Ann. Sci. École Norm. Sup. 8 (1975), 383-419.

[4] - 'Classification of injective factors, cases $\mathrm{II}_{1} \mathrm{II}_{\infty}, \mathrm{II}_{\lambda}, \lambda \neq 1$ ', Ann. of Math. (2) 104 (1976), 73-115.

[5] - 'On the classification of von Neumann algebras and their automorphisms', Symposia Mathematica, vol. 20 (INDAM, Rome, 1975) (Academic Press, London, 1976), pp. 435-478. 
[6] _ 'Periodic automorphisms of the hyperfinite factor of type $\mathrm{II}_{1}$ ', Acta Sci. Math. 39 (1977), $39-66$.

[7] A. Connes and M. Takesaki, 'The flow of weights on factors of type III', Tôhoku Math. J. 29 (1977), 473-555.

[8] U. Haagerup and E. Størmer, 'Pointwise inner automorphisms of von Neumann algebras with an appendix by C. Sutherland', J. Funct. Anal. 92 (1990), 177-201.

[9] V. F. R. Jones, Actions of finite groups on the hyperfinite type $\Pi_{1}$ factor, Mem. Amer. Math. Soc. 237 (Amer. Math. Soc., 1980).

[10] V. F. R. Jones and M. Takesaki, 'Actions of compact abelian groups on semifinite injective factors', Acta Math. 153 (1984), 213-258.

[11] Y. Katayama, C. E. Sutherland and M. Takesaki, 'The characteristic square of a factor and the cocycle conjugacy of discrete group actions on factors', Invent. Math. 132 (1998), 331-380.

[12] Y. Kawahigashi, 'One parameter automorphism groups of the hyperfinite type $\mathrm{II}_{1}$ factor', J. Operator Theory 25 (1991), 37-59.

[13] Y. Kawahigashi, C. E. Sutherland and M. Takesaki, 'The structure of the automorphism group of an injective factor and the cocycle conjugacy of discrete abeiian group actions', Acta Math. 169 (1992), 105-130.

[14] Y. Kawahigashi and M. Takesaki, 'Compact abelian group actions on injective factors', J. Funct. Anal. 105 (1992), 112-128.

[15] W. Krieger, 'On ergodic flows and the isomorphism of factors', Math. Ann. 223 (1976), 19-70.

[16] Y. Miwa, Remarks on tensor formulae of characteristic invariant and modular invariant for action on AFD factors (M.Sc. Thesis, Osaka Kyoiku University, 1999).

[17] A. Ocneanu, Actions of discrete amenable groups on factors, Lecture Notes in Math. 1138 (Springer, Berlin, 1985).

[18] Y. Sekine, 'Flows of weights of crossed products of type III factors by discrete groups', Publ. Res. Inst. Math. Sci. 26 (1990), 655-666.

[19] C. E. Sutherland and M. Takesaki, 'Actions of discrete amenable groups and groupoids on von Neumann algebras', Publ. Res. Inst. Math. Sci. 21 (1985), 1087-1120.

[20] - 'Actions of discrete amenable groups on injective factors of type $\mathrm{III}_{\lambda}, \lambda \neq 1$ ', Pacific $J$. Math. 137 (1989), 405-444.

[21] M. Takesaki, 'Duality for crossed products and the structure of von Neumann algebras of type III', Acta Math. 131 (1973), 249-310.

Division of Mathematical Sciences

Osaka-Kyoiku University

Kashiwara

Osaka 582

Japan

e-mail: katayama@cc.osaka-kyoiku.ac.jp 